\title{
0.2. Um Prelúdio De Vidas
}

\subsection{A Prelude Of Lives}

\section{Paula Guerra}

O De Vidas Artes sublinha as profundas mudanças que vão marcando o campo das artes no presente. As mudanças são múltiplas e os efeitos tão profundos que, em certos momentos, se torna difícil acompanhar e compreender os modos e direções em que se processam. Chamemos-lhe sociologia da arte ou sociologia da cultura (Heinich, 2010). Eduardo de la Fuente (2007) fala do surgimento de uma nova sociologia da arte, caraterizada por quatro pontos-chave: primeiro, uma reconsideração da relação entre sociologia e outras disciplinas; segundo, o surgimento de uma sociologia-arte (art-sociology) em oposição à sociologia da arte: terceiro, a aplicação de observações sobre arte a objetos não-artísticos, a coisas; quarto, a sociologia da arte passa a ser entendida como um facto social contingente.

A par do crescimento recente da sociologia das artes constatamos uma consequente especialização, bem como uma vontade de analisar e refutar as teorias de outras disciplinas académicas, como a filosofia ou a história da arte. Não deixa de ser um modo de marcar posição e, também é preciso dizê-lo, um compreensível complexo de inferioridade de quem chegou mais tarde ao jogo. Contudo, nos últimos anos, este dogmatismo perdeu força e as publicações mais recentes têm estabelecido aproximações muito interessantes às mais diversas disciplinas. Inglis e Hughson (2005) editaram um livro que englobava as mais diversas participações teóricas como a semiótica, história da arte, arquitetura e estudos culturais. Se Bourdieu continua a ser o principal referente teórico, o anti-elitismo de Becker (2010) é prevalente, isto é, a ideia desmistificadora sobre o trabalho artístico, sobre o artista e mesmo sobre o facto de o estudo sobre a arte não ser um simples exercício académico abstrato que interesse apenas a uma reduzida plateia de investigadores. Estes autores parecem afins de Alexis de Tocqueville quando este afirmava que "[n]ada é mais improdutivo para o espírito humano do que uma ideia abstracta" (Tocqueville, 2007: 756).

A única forma de o evitar, e obter-se assim um futuro menos dogmático, é se os sociólogos e as sociólogas começarem a relativizar, historicizar e 
colocar a nu todas as suposições dadas como óbvias. DeNora (2003) seguindo esta linha de pensamento, considera que o melhor seria aplicar um novo termo, como art-sociology, em que a arte não seja um mero objeto, mas sim uma força ativa na vida social das pessoas. Um afastamento da tal "ideia abstracta" que Tocqueville nos falava e que tão cara era a autores na linha de Adorno - que podem ser caracterizados por uma sociologia da arte ao nível de generalização. É, portanto, necessário tratar a arte como arte. Uma sociologia onde não existam categorias a priori, em que a arte não seja apenas o resultado de determinadas forças sociais. Uma perspetiva que apenas leva a olvidar as propriedades ativas das artes, bem como o seu potencial na vida das pessoas.

Sam de Boise (2016) - na linha de Antoine Hennion (2010) -, considera que nas abordagens bourdeusianas raramente existe espaço para a própria arte. A forma como a arte é construída, lida e recebida é extremamente importante para que um investigador consiga apreender o porquê de certas pessoas gostarem e outras não. Na sua perspetiva, falta a perceção que a arte serve, de múltiplas maneiras, de motor de desenvolvimento comunitário, em lutas contra o racismo, sexismo, exploração, etc., e que em muitos casos alcançam resultados positivos. Nessa linha, Eyerman e McCormick (2006) referem que através da arte surgem novas identidades e práticas sociais. Através da arte é possível criar espaços para experimentação com projetos sociais, políticos e estéticos. Veja-se o estudo de Bergh (2007) que analisou como, por exemplo, a música pode deter um papel determinante para resolução de conflitos no Sudão.

Para ter essa sensibilidade é preciso uma clara mudança a nível empírico e analítico: passar de um enfoque no gosto - entendido enquanto aquisição socializada - para um enfoque na forma como as pessoas interagem com a arte (Prior, 2013). Nesta moldura enquadra-se também a linha de investigação relativa ao ethos do-it-yourself e de como remete para uma nova e diferente relação com a arte (Bennett \& Guerra, 2019; Guerra, 2018). A obsessão com os inquéritos relativos ao gosto artístico teve o efeito nefasto de os inquiridos já saberem o que o sociólogo espera deles. Ora, isto leva a respostas cada vez mais previsíveis. Por isso, Hennion fala da necessidade de "des-sociologizar os amadores para que eles possam falar não apenas dos seus determinismos mas das suas formas de atuar" (Hennion, 2010: 27-28). 
Se é verdade que os resultados que Pierre Bourdieu (2010) obteve sobre o processo de distinção social já não se aplicam à sociedade atual, isso é mais pelo facto de Bourdieu ter escrito na década de 1970 e não por erros na sua metodologia. É necessário uma reactualização. Têm sido vários os estudos sobre o capital cultural, com resultados deveras interessantes. Qual a razão do enfoque no capital cultural? Para DiMaggio (1982), a razão é simples: as artes são a forma cultural de reconhecimento mais prestigiada no mundo ocidental. Em qualquer grupo pode existir formas de conhecimento prestigiantes. Por outro lado, conhecimento artístico, é, em geral, o mais prestigiante e um forte indicador de um capital cultural. E apesar de nas últimas décadas vários estudos empíricos terem suportado as premissas postuladas por Bourdieu, autores como DiMaggio e Mukhtar (2004) falam de um declínio da posição da arte como capital cultural. E apontam várias razões para tal declínio: primeiro, a ubiquidade da cultura popular, que obviou a que os até então gatekeepers culturais, como universidades e instituições culturais, mantivessem a sua centralidade cultural (Warde, et al., 1999); segundo, a ideia prevalecente que a alta cultura está a esboroar-se, ocorrendo uma des-institucionalização, fruto quer do multiculturalismo quer dos próprios artistas, que recusam as barreiras entre cultura popular e séria (DiMaggio \& Mukhtar, 2004); terceiro, o prestígio cultural advém atualmente da familiaridade com múltiplas formas artísticas. O que Peterson e Kern (1996) apelida de omnívoros, seriam, então, os novos detentores do capital cultural e seriam agentes capacitados para se movimentarem entre cultura popular e alta (Friedman et al, 2015).

Trata-se de uma forma de capital cultural emergente. Existe uma diferença na relação com o cânone artístico e, por outro lado, existe uma valorização do novo por partes dos mais jovens. Assim, as diferenças de gosto cultural, em vez de apenas serem analisadas a partir das diferenças classistas, devem também atender a diferenças geracionais. É a própria cultura dominante que está a passar por uma processo de transformação, ainda que a longo prazo. Temos de ter em atenção que estas novas culturas dominantes abandonam as barreiras nacionais ou europeias, o que origina formas de capital cultural cosmopolita que propiciam a rutura com uma visão da cultura assente numa tradição nacional, intimamente ligado ao conceito de Estado-Nação. Este capital cultural emergente não deve ser dissociado do processo de informalização (Wouters, 2004, 2007). Uma das 
características marcantes do século $X X$ foi 0 relaxamento dos constrangimentos emocionais e comportamentais nas interações sociais, sendo de relevar os festivais (Guerra, 2016, 2017). Para Cas Wouters (2004), tal deveu-se à crescente influência das camadas mais baixas da sociedade, os seus modos comportamentais, mais informais, acabaram por ser incorporados por toda a sociedade. Os contributos incluídos neste livro englobam e centralizam esta reticularidade e obliquidade das pesquisas em torno das artes.

Posto isto, consideramos que neste livro De Vidas Artes podemos visualizar todas as profundas mudanças que aqui elencámos. Dividido em quatro partes, pretende-se na primeira - Partes das Artes - o recorte sóciohistórico e sociológico (incluindo o pensamento sobre o próprio recorte) e o estudo de alguns dos atores e agências fundamentais na arte contemporânea: jovens artistas, colecionadores, coletivos multidisciplinares, galeristas, mediadores, associações culturais, eventos. Assim, o primeiro capítulo, "Welcome to Inhotim, fruto de mecenato feito com renda de mineração!": um ensaio sobre colecionismo trata alguns aspectos do colecionismo particular de arte contemporânea. Dayana Zdebsky de Cordova pensa através das diferentes imagens-seres do colecionador brasileiro Bernardo Paz e do Instituto Inhotim. É nesta linha que desenvolve uma experiência de pesquisa etnográfica sobre colecionismo e o mercado brasileiro de arte contemporânea com extensões das reflexões sobre Paz e Inhotim para certas lógicas do colecionismo de arte contemporânea. Continuando o recorte da primeira parte, Leonardo Bertolossi elabora o segundo capítulo e analisa a antropologia das razões simbólicas que motivaram a vida das galerias e o ofício de galeristas no Brasil. Da Arte de Vender Arte: Uma Antropologia das Galerias e Galeristas Brasileiros percorre assim alguns episódios, personagens e aspectos fundamentais das suas histórias para destacar a biografia do mercado primário de arte contemporânea. A primeira parte segue com o contributo de Diego Soares Rebouças. Na sua exposição, que denominou Amontoado de destroços: a trajetória de Miguel Rio Branco via imagens e montagens artísticas, o autor parte da vida e obra do artista Miguel Rio Branco para questionar as elaborações de arte contemporânea e o papel das imagens, sobretudo em instalações artísticas, que desafiam aquilo que nas discussões académicas nos campos da arte, da imagem, da história, da sociologia (da arte) se tornou 
um lugar-comum: o tramento das clivagens e bifurcações nas elaborações discursivas sobre imagens artísticas no contexto das rupturas e polémicas causadas pela arte contemporânea. O quarto capítulo da primeira parte intitula-se Janelas para o mundo: A Mostra Internacional de Cinema de São Paulo e o Festival de Cinema do Rio como palco para a festa de seus públicos e foi escrito por Bianca Salles Pires. Nele são vincados os aspectos sóciohistóricos de dois grandes eventos cinematográficos do Brasil, a Mostra Internacional de Cinema de São Paulo e o Festival de Cinema do Rio de Janeiro. Bianca aborda os eventos de cinema e a participação dos públicos contemporâneos a partir dos dados reunidos entre os anos de 2015 e 2017 durante a etnografia realizada em três edições da Mostra Internacional de Cinema de São Paulo e do Festival de Cinema do Rio. A sua análise procura contribuir, à luz da bibliografia internacional acerca dos festivais, para o pensamento reflexivo das cinefilias de mostras de cinema no Brasil. O quinto texto Coletivos de arte: notas sobre memória, política e autoria constitui o espaço que a autora Ana Carolina Freire Accorsi Miranda dedica a questões que permeiam a historiografia dos coletivos de arte no Brasil, procurando mostrar as possíveis contaminações desses grupos noutras esferas do mundo da arte, tais como a curadoria e a gestão de museus. Para o que se propõe, inicia uma retrospectiva das principais fases que marcaram aquele percurso, destaca a relação entre as transformações urbanas recentes e o crescimento dos coletivos, discute o contexto político e sua contaminação nesta área da arte e, por fim, analisa a autoria colaborativa na sociedade contemporânea. O sexto e último capítulo desta primeira parte, Procuram-se (Jovens) Artistas, da autoria de Guilherme Marcondes, reflecte os processos de legitimação dos jovens artistas selecionados pelo sistema da arte contemporânea e que, ao participarem assim de exposições nas mais variadas e legitimadas instituições artísticas, iniciam o processo de construção de suas carreias e eventual legitimação.

A segunda parte do livro - Limites das Artes - inclui pesquisas sobre a vigência e as precariedades de fronteiras, de demarcações, de canonizações e de definições entre modalidades, mediações, linguagens e atores das artes. Assim iniciamos com a perspectiva social de Voica Puscasiu na exploração de algumas maneiras pelas quais as práticas artísticas surgiram nas ruas e, embora o foco esteja principalmente nas artes visuais, o texto Art on the Streets: Past and Present Practices também observa a relação em 
constante evolução das práticas mencionadas com as instituições, tanto das artes estabelecidas quanto das pertencentes à cidade, assim como as políticas que as medeiam. Em seguida, o capítulo Literatura de cordel no ciberespaço: As tecnologias digitais no processo de escrita de Rafael da Silva da Cunha propõe-nos a compreensão de como a mediação das tecnologias digitais influenciam o processo de escrita da literatura de cordel, podendo potencializar novas experiências cognitivas. A partir da etnografia virtual como método de pesquisa, o autor constata que a literatura de cordel vive um momento de transgressão do suporte textual onde os cordelistas adotam estratégias de criação específicas para o ambiente virtual. A estrutura da segunda parte recebe no terceiro capítulo os aportes teóricos sociológicos e da história da arte de Tálisson Melo de Souza. Em Cartografias estéticas entre mediação e esfera pública: como a 'arte latino-americana' acontece? lemos como o autor estuda a atuação de intermediários no universo artístico, e as exposições como eventos/contextos em que as obras de arte se apresentam na esfera pública, compondo sua dimensão estética. A partir de algumas exposições e outras iniciativas de agentes intermediários baseados nos Estados Unidos, Tálisson refere-nos exemplos de como se constitui a categoria 'arte latino-americana' em diferentes períodos. Sem perder o foco da segunda parte do presente livro, seguimos o quarto dos seus capítulos. Elenise Andrade explora em Cidades, gestos, imagens em provoc-ações o seu projeto de pós-doutoramento "Provoc-ações: (des)ocupar imagens, (des)enquadrar escritas, perambular por Bahias e Portos" realizado na Faculdade de Letras da Universidade do Porto e que envolveu três instituições escolares portuguesas. Como sublinha a autora, neste projeto procuraram-se aproximações entre educação e conceitos da filosofia da diferença pela via das visualidades. Deste modo, presenciaremos as Oficinas entendidas como momentos de fazer/pensar imagens, algo que vai além dos procedimentos analíticos da representação, explicação e registo da natureza das mesmas. Sara de Andrade assina o texto Entre a arte e o político: mediação e o apagamento dos limites do campo artístico, o penúltimo desta parte que, partindo do exemplo prático da repercussão negativa que levou ao encerramento da exposição "Queermuseu - Cartografias da diferença na arte brasileira", procura entender como as reações à arte, e a arte em si, são pervasivas a fatores como o contexto político e social, as mediações a que a arte é exposta ou as discussões e sentidos produzidos pelos públicos na relação entre espectador e obra. Concluímos os Limites 
das Artes com a contribuição de Jessica Gogan. A reflexão que desenvolve ao longo do seu Que se fosse para o mundo: Por uma curadoria ao avesso é uma pesquisa em progresso, uma extensão da sua tese de doutoramento, para afirmar a conceptualização de uma teoria/prática do que designou "uma curadoria ao avesso", ancorada numa abordagem ecossistémica do cuidado na contemporaneidade ou, ainda, em perspectivas críticas e clínicas que apontam para a proximidade como posicionamento ético e para práticas contemporâneas que experimentam diversos dispositivos de arte-ação.

Artes dos Limites é o tema da terceira parte de De Vidas Artes. O fio condutor é a intertextualidade das artes que, em última análise, revela a arte onde ela não estaria, ou onde não suporíamos que estivesse; eis a arte escondida, a arte misturada. Iniciamos essa parte do livro com a música, seguindo o trabalho que Sofia Sousa intitulou Bairro (d)e Música! Apropriações, Musicalidades e Simbologias do Bairro do Cerco do Porto. Apoiando-se no conceito de música enquanto motivação e motor de impulsão de inclusão social, a autora recorre a uma análise de pendor qualitativo do bairro do Cerco do Porto dando a conhecer processos de investigação que deram voz a indivíduos, problemas, conceitos e significações. Susana Januário é responsável pela teorização que compreende o segundo capítulo desta parte. Em Manifestações artísticas alternativas ou a face visível de um Portugal contemporâneo: da problematização dialógica de conceitos ao mapeamento de um subcampo híbrido seguimos a investigação da autora nos moldes duma compreensão de fenómenos genericamente tidos como alternativos/underground. A emergência destas manifestações artísticas assenta em lógicas do-it-yourself com uma base plural e experimental e pode distribuir-se segundo um mapeamento que procede da análise de conteúdos disponibilizados por dispositivos mediáticos. O terceiro texto é escrito por Ana Martins e segue as mitologias que envolvem a (sub)cultura rock portuguesa contemporânea. Estigmas, rotulações e apropriações no rock português (1960-2020) avança no sentido de desmontar os possíveis rótulos associados àquela subcultura, de modo a evidenciar os possíveis mecanismos de dominação social e, enfim, proceder a uma efetiva construção da realidade que contrapõe alternativas ao clássico epíteto de desviante associado a atitudes e comportamentos da (sub)cultura rock. Tanja Baudoin conduz-nos, de seguida, pelas linhas de Limites móveis. Sobre dispositivos de enquadramento na obra 'eu-você' de Ricardo Basbaum. Com o seu ponto 
de partida num estudo da obra do artista Ricardo Basbaum, pensa-se de que maneiras uma obra de arte contemporânea pode moldar ativamente estruturas para sua própria produção e receção, atravessando limites e rompendo molduras que culminam numa nova relação com o público - este abandona a atitude contemplativa e passa a ter outra mais direta e ativa. Em vários pontos, a autora assume a questão sobre "os limites entre arte e vida" definindo a sua posição face a estes conceitos. Seguimos a moldura da terceira parte em $O$ artivismo de Hélio Rôla: Do pincel ao pixel, o capítulo quinto assinado por Flávia Fernanda Fernandes. O texto de Flávia tem como propósito realizar uma reflexão sobre artivismo a partir da obra e do percurso do artista visual Hélio Rôla, o exemplo de um artivismo que transita da pintura mural na década de 70 para a actualidade das redes sociais com o intuito de intervir no cotidiano, produzindo não só obras, mas também testemunhos do seu tempo. Clarisse Martins Monteiro com o capítulo intitulado Mediações em Pequenas Vozes: práticas distintas no contato com a arte fecha esta parte. O presente trabalho tem como objetivo ampliar o entendimento sobre o conceito de mediações e contribuir para as discussões no campo da arte e sociologia da arte, através da análise do processo de educação em artes e da produção artística de jovens pertencentes às classes populares do Centro do Rio de Janeiro, participantes do projeto social Pequenas Vozes do Carmelo.

Do caminho que delineámos falta percorrer a quarta e última parte. Artes Ilimitadas evidencia a necessidade do questionamento de posições tácitas das teorias da Arte e afirma a relevância da renovação da sociologia da arte para a análise dos movimentos artísticos e para a própria possibilidade de distinção da arte da não arte. Foca ainda a inescapável autoreflexividade que a sociologia, como qualquer outra área de tematização humana, deve empreender quanto aos cânones e normatividades que a fundam. Sob esta égide temática reúnem-se nos seis capítulos constituintes trabalhos que exploram a ideia de artes ilimitadas. $O$ primeiro contributo é de Arthur Arantes Souza. Em Glauber Rocha: campo de estudos sobre um cineasta procurou estabelecer as representações compartilhadas entre pesquisadores do diretor Glauber Rocha e explicar as suas causas, com recurso a técnicas de análise de conteúdo para investigar as regularidades nos discursos das teses e, ainda, a metodologia bibliométrica. No segundo capítulo lemos de Thiago Pereira Alberto o texto Cultura pop e nostalgia na 
contemporaneidade: apontamentos iniciais sobre arquivo, memória e o YouTube como um espaço heterotópico. O YouTube, enquanto possibilidade de arquivamento infindável, é aqui escalpelizado como um campo de análise notável que expõe a relação entre arquivo e memória como um traço formativo para a noção de cultura pop na contemporaneidade. Deste modo, apercebemo-nos de que estamos perante um importante fenômeno comunicacional centrado na ideia de nostalgia e consumo retro. Chegamos ao terceiro capítulo: Do ethos à praxis. Carreiras DIY na cena musical independente em Portugal, onde Ana Oliveira trata a profissionalização na música, explorando as relações entre independência, carreiras DIY e sustentabilidade económica. Centra-se na cena musical independente das áreas metropolitanas de Lisboa e Porto e, com base em entrevistas semiestruturadas, Ana procura elucidar-nos sobre um entendimento do que é hoje ser músico em Portugal e que estratégias são mobilizadas pelos artistas na gestão das suas carreiras. Tânia Moreira conduz-nos pelos tons e relevos da música de dança eletrónica (MDE) e o psytrance. Neste capítulo, A Arte Elétrica de Ser Português, a autora analisa resumidamente o surgimento da MDE, expõem a teoria que estruturou o seu estudo e, em seguida, apresenta o retrato global da produção científica da MDE. Das principais características do psytrance português parte para considerações sobre os fluxos culturais que ocorrem na globalização, bem como para a importância de uma renovação teórica no campo dos cultural studies. Ainda num fio musical e musicado o texto seguinte é de Dulce Mazer com o título "Rapper, musa, música, mulher". Empoderamento feminino e imbricações do regionalismo gaúcho na cultura hip-hop. Na sua estrutura veremos como os métodos do estudo (a observação etnográfica e a pesquisa documental na Região Metropolitana de Porto Alegre) reflectem o modo como as rappers têm alterado as suas práticas socioculturais na cultura hip-hop, considerando uma hibridação com o regionalismo gaúcho. Terminamos o conteúdo das Artes llimitadas com o capítulo dedicado a Arto Lindsay, um multiartista, como refere Pérola Mathias, a autora de Arto Lindsay: um artista contemporâneo na música brasileira. É-nos proposto olhar esta obra plural como exemplar da importância de Arto Lindsay na transformação da música popular no Brasil através de suas criações, mediações e rede de relações. A partir da trajetória e obra de Lindsay, Pérola Mathias pondera a possibilidade de pensar a configuração da arte na contemporaneidadade. 
Nos tempos recentes, as artes têm vindo a ganhar um papel de relevo - pelo menos mediático - como estratégias de planeamento em prol da regeneração urbana, da inclusão de populações desfavorecidas, de combate a uma sociedade cada vez mais desigual e à pobreza daí decorrente, de empoderamento de minorias, de credenciação de mulheres e da população LGBTQI+, de fomento de uma "economia criativa" e diversas possibilidades de exercício de trabalho e obtenção de profissão num contexto de precariedade económica e risco social. Portanto, as artes têm-se prefigurado dentro de uma lógica de panaceia universal contra todos os males que atravessam a humanidade, em parte porque muitas das iniciativas que as colocam no centro de ação são sobretudo de natureza retórica e mediática e não propriamente política.

Não obstante, não podemos deixar de reconhecer as artes e a cultura no coração dos processos de reconstrução identitários contemporâneos de povos e de nações. Um qualquer olhar que oriente a sua atenção sobre a situação cultural das últimas décadas notará a proliferação de produções e eventos artísticos de índole estética que não se compatibilizam com as lógicas de produção, canonização, mediação e fruição artísticas anteriores. Tal pode exprimir, numa primária observação, que o cenário presenteado pelas artes atuais se modificou de tal forma que as estruturas e esquemas mentais não têm capacidade para as explicar e compreender, representando-as como estranhas, caóticas, heterógenas e até não arte. Mas a verdade é que um olhar mais atento leva-nos a outras questões. Emergem, por vez, subterraneamente, disfarçadamente, formas, produtos, atores, eventos, plataformas artísticas plurais, "omnívoras" para sermos fiéis a Peterson (Peterson e Kern, 1996).

Tornam-se notórias, neste contexto, as apreciações acerca da dialética entre estetização do quotidiano e quotidianização das estéticas - de facto, o espaço urbano global é simultaneamente constituído e constitutivo desta operação. A cidade global é duplamente mediadora por referência a estas dinâmicas culturais: o meio em que elas se geram e tomam forma é também o meio pelo qual elas se realizam, fornecendo o conjunto infraestrutural que possibilita a sua emergência e oferecendo os lugares e os agentes que cultivam estas artes. Neste livro conflui com tudo isto: com as contaminações das artes com as vidas, com os estilos de vida, com alta cultura, com a cultura de massas, com as culturas populares, com a 
moda, com o turismo, com as profissões, com a precariedade, com o do-ityourself.

Embora, em geral, bem estudadas em campos já assentes nas ciências sociais - como o da sociologia da arte - imaginamos poder estabelecer novos contatos, cruzamentos e aproximações dessas temáticas por meio do trabalho de jovens pesquisadores que, utilizando as perguntas e as dúvidas que teorias sobre a arte e a vida social suscitam, trazem redefinidos e renovados pensamentos que interessam a todas e a todos que continuam devidamente envolvidos e perplexos e refletindo sobre a arte. Esperamos assim que De Vidas Artes vos proporcione boas leituras e novas inspirações.

\section{Referências Bibliográficas}

Acord, S. K. \& DeNora, T. (2008). Culture and the arts: from art worlds to arts-in-action. The ANNALS of the American Academy of Political and Social Science, 619(1), pp. 223237.

Becker, H. S. (2010). Mundos da arte. Lisboa: Livros Horizonte.

Bennett, A. \& Guerra, P. (Eds.) (2019). DIY cultures and underground music scenes. Collection Routledge Advances in Sociology. Oxford: Routledge.

Bergh, A. (2007). I'd like to teach the world to sing: Music and conflict transformation. Musicae Scientiae, 11(2), pp. 141-57.

Bourdieu, P. (2010). A distinção. Uma crítica social da faculdade do juízo. Lisboa: Edições 70.

de Boise, S. (2016). Post-Bourdieusian moments and methods in music sociology: Toward a critical, practice-based approach. Cultural Sociology, 10(2), pp. 178-194.

DeNora, T. (2000). Music in everyday life. Cambridge: Cambridge University Press.

DeNora, T. (2003). After Adorno: Rethinking music sociology. Cambridge: Cambridge University Press.

DeNora, T. (2011). Music-in-action: essays in sonic ecology. Farnham: Ashgate.

DeNora, T. (2013). Music Asylums: Wellbeing through music in everyday life. Farnham: Ashgate.

DiMaggio, P. (1982). Cultural capital and school success: the impact of status culture participation on the grades of U.S. high school students. American Sociological Review, 47(2), pp. 189-201.

DiMagio, P. \& Mukhtar, T. (2004). Arts participation as cultural capital in the United States, 1982-2002: Signs of decline? Poetics, 32(2), pp. 169-194. 
Eyerman, R. \& McCormick, L. (Eds.) (2006). Myth, meaning, and performance: Toward a new cultural sociology of the arts. Boulder, CO: Paradigm.

Fuente, E. de la (2007). The "new sociology of art": putting art back into social science approaches to the arts. Cultural Sociology, 1(3), pp. 409-425.

Gadamer, H.-G. (2013). Truth and method. London/New York: Bloomsbury.

Guerra, P. (2016). "From the night and the light, all festivals are golden": The festivalization of culture in the late modernity. In Guerra, P. \& Costa, P. (Eds.), Redefining art worlds in the late modernity (pp. 39-67). Porto: University of Porto.

Guerra, P. (2017). A revolução do festival: um percurso pela agenda dos festivais pop rock portugueses na última década. In de Almeida P., Vitor \& de Almeida, L. (Eds.), Circuitos urbanos, palcos midiáticos: Perspetivas culturais da música ao vivo (pp. 2953). Maceió: Edufal.

Guerra, P. (2018). Raw Power: Punk, DIY and underground cultures as spaces of resistance in contemporary Portugal. Cultural Sociology, 12(2), pp. 241-259.

Heinich, N. (2010). What does 'sociology of culture' mean? Notes on a few transcultural misunderstandings. Cultural Sociology, 4(2), pp. 257-265.

Hennion, A. (2010). Loving music: From a sociology of mediation to a pragmatics of taste. Scientific Journal of Media Education, 17(34), pp. 25-33.

Inglis, D. \& Hughson, J. (Eds.) (2005). The sociology of art: Ways of seeing. London: Palgrave.

Peterson, R. A.; Roger M. (1996). Changing highbrow taste: from snob to omnivore. American Sociological Review, 61(5), pp. 900-907.

Silva, A. S. \& Guerra, P. (2015). As palavras do punk. Lisboa: Alêtheia.

Tocqueville, A. de (2007). Da democracia na América. Estoril: Principia.

Warde, A., Martens, L. \& Olsen, W. (1999). Consumption, the problem of variety cultural omnivorousness. social distinction, dining out. Sociology, 33(1), pp. 105-127.

Wood, N., Duffy, M. \& Smith, Susan J. (2007). The art of doing (geographies of) music. Environment and planning. Society and Space, 25(5), pp. 867-889.

Wouters, C. (2004). Sex and manners: Female emancipation in the west 1890-2000. London: Sage.

Wouters, C. (2007). Informalization: Manners and emotions since 1890. London: Sage. 